\title{
Performance study of node placement in sensor networks
}

\author{
Mika ISHIZUKA and Masaki AIDA \\ NTT Information Sharing Platform Labs, NTT Corporation \\ 3-9-11, Midori-Cho Musashino-Shi Tokyo 180-8585 Japan \\ \{ishizuka.mika, aida.masaki\}@lab.ntt.co.jp
}

\begin{abstract}
The expectations for sensor networks are growing. One of the most important issues in sensor networks is to evaluate the fault tolerance and built technology to improve $i t$, because sensor nodes are prone to fail and have limited power capacity. So far, research on the fault tolerance has focused on battery exhaustion. Since placement affects whether there are nodes that can sense and routes to the base station, placement of sensor nodes is also important. However, there has been little research on the placement of sensor nodes and all of it is based on deterministic placement, which is not realistic when many sensor nodes are placed in a large area. In such a situation, stochastic placement is needed. Therefore, this paper evaluates the tolerance against both random failure and battery exhaustion from the viewpoint of stochastic node placement.
\end{abstract}

\section{Introduction}

Recent advances in electronics such as power saving LSIs have led to small sensors with communication capability. Since a sensor network composed of these sensor nodes enables wide area and large-scale information gathering, expectations for sensor networks are growing.

Although a sensor network is a kind of ad hoc network, it differs in some ways and has new issues [1]. One issue is to evaluate the fault tolerance and build technology that can improve it, because sensor nodes are prone to fail and have limited power capacity.

So far, research on fault tolerance has focused on battery exhaustion. The main research topics are the power saving mode of the MAC protocol $[9,11,10]$ and energy efficient route selection [4, 3, 7]. Since placement affects whether there are nodes that can sense and routes to the base station, the placement of sensor nodes is also important. However, there has been little research on the placement of sensor nodes and all of it is based on deterministic placement $[5,2]$, which is not realistic when many sensor nodes are placed in a large area. In such a situation, stochastic placement is needed.

Therefore, this paper evaluates the fault tolerance from the viewpoint of stochastic node placement. Moreover, this paper deals with the tolerance against both random failure and battery exhaustion. This is because we consider that tolerance against random failure is also important since sensor nodes are prone to fail.

\section{Background}

\subsection{Communication Model}

In this paper, we consider applications where we cannot know the location of the target in advance (e.g. monitoring a vehicle in a forest). A target is generated in region $A$. A sensor node can explore a target only if it is in sensing range of the node. A sensor node can transmit data to or receive data from other sensor nodes within the radio transmission range. Generally, the sensing range is much smaller than the radio transmission range. When a target is generated, all the sensor nodes that have it within their sensing ranges send the sensory information to the base station during period $D$. Here, the data sending rate is so small that no data collisions occur. Routes are selected based on the minimum hop strategy. An example of the sensing process is illustrated in Fig. 1.

A sensor node consumes its battery energy to transmit $\left(E_{t}[\mathrm{~J} / \mathrm{bit}]\right)$ and to receive bits $\left(E_{r}[\mathrm{~J} / \mathrm{bit}]\right)$. When a sensor node exhausts its battery energy, all the functions of the sensor node stop and an alternative route is selected for the remaining information.

\subsection{Placement of sensor nodes}

When a lot of sensor nodes are placed in a large area, it is not realistic for someone to place them all at predetermined positions. Instead, stochastic placement is needed. In this paper, we consider three typical types of stochastic sensor-placement. 


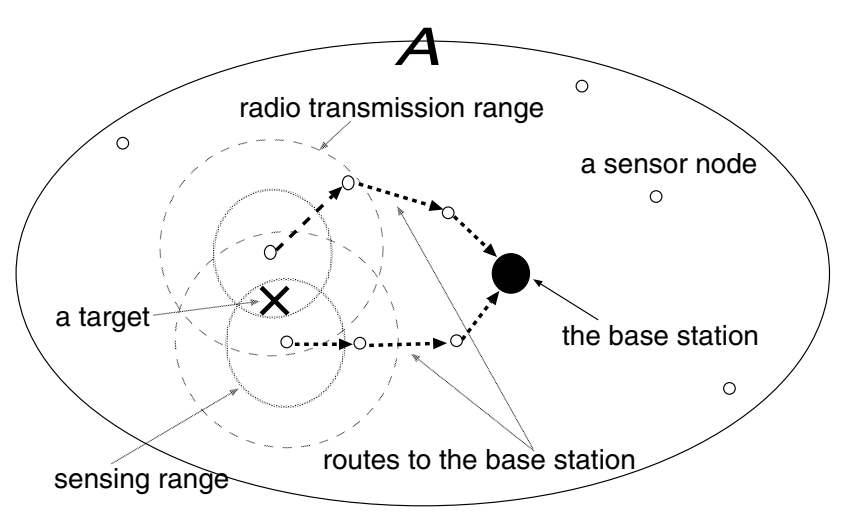

Figure 1. Example of sensing.

Simple diffusion The simplest way to distribute sensornodes is to scatter them from the air. We call this simple diffusion. Since all the information must reach the base station, we center the distribution on the base station.

If weight of sensor nodes is light, they will have high air resistance. This randomizes their placement, which can be described by diffusion equation. Since solution of diffusion equation forms the two-dimensional normal distribution, $f(\boldsymbol{x})$, the probability density function (p.d.f) of sensor-position $\boldsymbol{x} \in R^{2}$ is ${ }^{1}$

$$
\begin{aligned}
& f(\boldsymbol{x})=\frac{1}{2 \pi \sigma^{2}} h(\|\boldsymbol{x}-\boldsymbol{c}\|), \\
& h(r) \triangleq \exp \left(-\frac{r^{2}}{2 \sigma^{2}}\right) .
\end{aligned}
$$

In (1), $c \in R^{2}$ is the position at ground which is just under the point where sensor nodes are scattered and $\sigma^{2}$ is the variance of the distribution. The variance is determined by various factors (e.g., shape or weight of sensors, or the hight at which sensors are released).

An example of simple diffusion is illustrated in Fig. 2. Here, the number of sensor nodes is 400 and the variance is set so that $99 \%$ of them may be placed within $500[\mathrm{~m}]$ of the base station.

Constant placement In many papers, sensor nodes are placed so that their density is constant. We call this constant placement. The p.d.f of sensor-positions is

$$
f(\boldsymbol{x})=\frac{1}{|A|} .
$$

1 The probability of a sensor being within region $A=\left\{X_{1} \leq\right.$ $\left.x_{1}, X_{2} \leq x_{2}\right\}$ can be written by using p.d.f as follows: $P\left\{X_{1} \leq\right.$ $\left.x_{1}, X_{2} \leq x_{2}\right\}=\int_{-\infty}^{x_{2}} \int_{-\infty}^{x_{1}} f\left(x_{1}, x_{1}\right) d x_{1} d x_{2}$.
An example is illustrated in Fig. 3, where the number of sensor nodes is 400 and they are within 500 [m] of the base station.

R-random placement Finally, we propose a new method called the R-random placement. Sensor nodes are uniformly scattered in terms of the radius and angular direction from the center, which coincides with the base station.

When all the sensor nodes are within range $R$ of the base station, the p.d.f of sensor-positions in polarcoordinates $(r, \theta)$ is

$$
f(r, \theta)=\frac{1}{2 \pi R}, 0 \leq r \leq R, 0 \leq \theta<2 \pi .(4)
$$

In (4), we set the base station to the origin without losing generality. An example of R-random placement is illustrated in Fig. 4, where the number of sensor nodes is 400 and all of them are within 500 [m] of the base station.

Note that density of sensor nodes nearer the base station is higher in R-random placement, though p.d.f of sensor-positions does not depend on radius and angle. Actually, $\rho\left(r_{1}, \theta_{1}\right)$, the density of sensor node at position $\left(r_{1}, \theta_{1}\right)$, is given as follows:

Let $S(a \leq r \leq b, c \leq \theta \leq d)$ be the area of the region $X=\{a \leq r \leq b, c \leq \theta \leq d\}$, then

$$
\begin{aligned}
& \rho\left(r_{1}, \theta_{1}\right)= \\
& \lim _{\delta r \rightarrow 0} \lim _{\delta \theta \rightarrow 0} \frac{\int_{r_{1}}^{r_{1}+\delta r} \int_{\theta_{1}}^{\theta_{1}+\delta \theta} f(r, \theta) d \theta d r}{S\left(r_{1} \leq r \leq r_{1}+\delta r, \theta_{1} \leq \theta \leq \theta_{1}+\delta \theta\right)} \\
& =\lim _{\delta r \rightarrow 0} \lim _{\delta \theta \rightarrow 0} \frac{1}{\pi R\left(2 r_{1}+\delta r\right)}=\frac{1}{2 \pi R r_{1}} .
\end{aligned}
$$

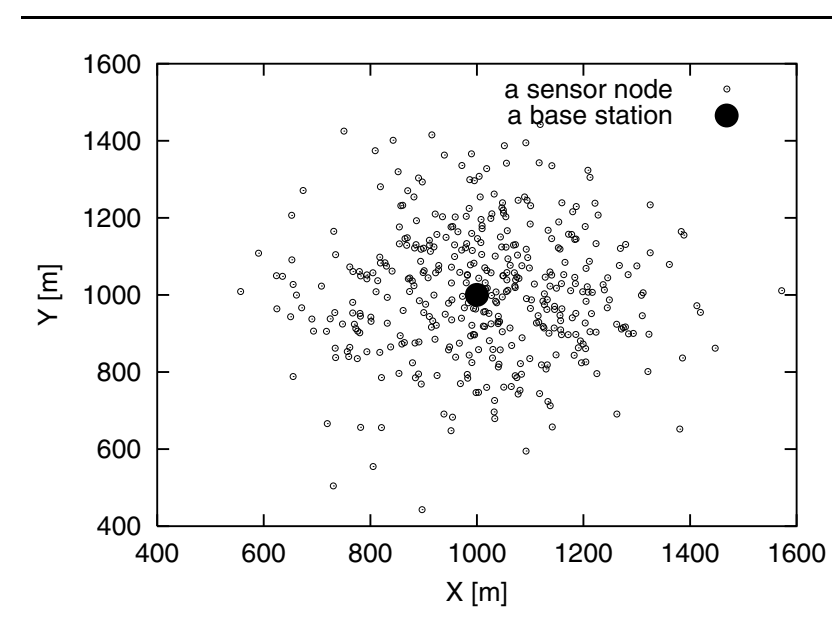

Figure 2. Example of simple diffusion. 


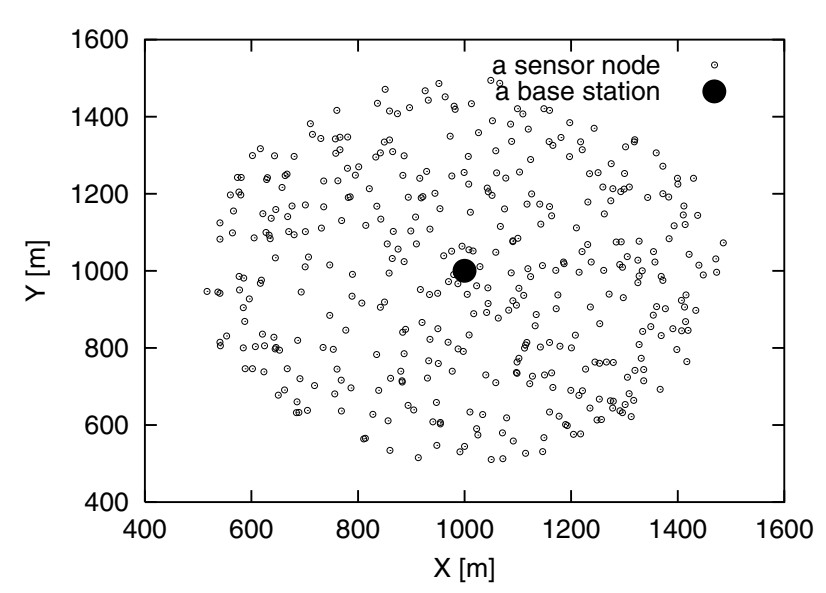

Figure 3. Example of constant placement.

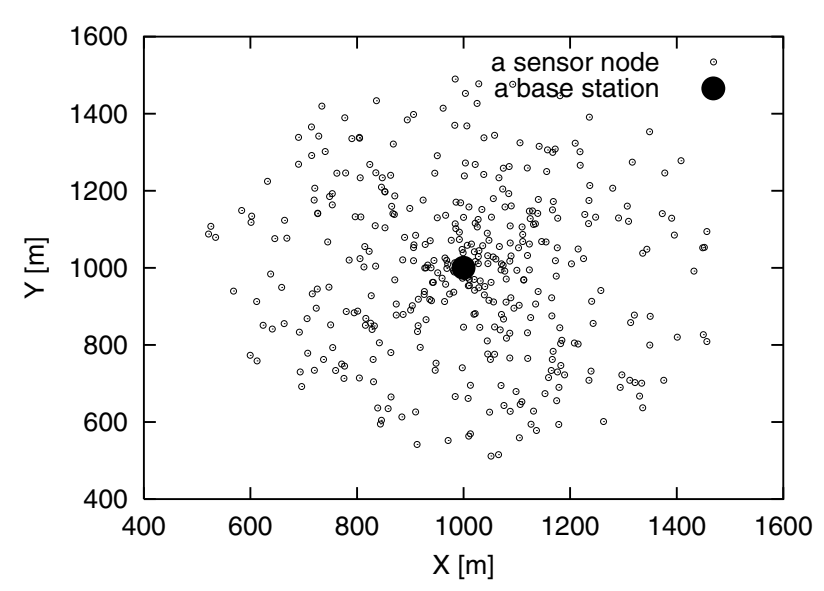

Figure 4. Example of R-random placement.

Note that the normal distribution can be used as a basis function. Therefore, any p.d.f of sensor-position can be approximated as a superposition of normal distributions as follows [8]:

$$
f(\boldsymbol{x})=\sum_{i=1}^{N} p_{i} h\left(\left\|\boldsymbol{x}-\boldsymbol{c}_{\boldsymbol{i}}\right\|\right)
$$

That is, repeated simple diffusion can lead to any stochastic sensor-placements.

\section{Simulation Settings}

\subsection{Setting of targets}

The sensing targets were randomly generated within region $A$, a circle with a radius of $500[\mathrm{~m}]$ centered on the base station.

For the evaluation of tolerance against battery exhaustion, we set the target sensing period to be exponentially distributed with an average of 72 [min].

\subsection{Setting of sensor nodes}

The number of sensor nodes was 250 . For constant and R-random placement, all the sensor nodes were placed in region $A$. For simple diffusion, the variance was set so that $99 \%$ of sensor nodes would be placed within region $A$.

The sensing range was set to $60[\mathrm{~m}]$ and the the radio transmission range is to $100[\mathrm{~m}]$. The data sending rate was 1.1 [kbps], which is enough low to avoid collisions. Energy consumption was $3.3 \mathrm{e}-07$ [J/bit] to transmit bits and $1.9 \mathrm{e}-$ 07 [J/bit] to receive [6]. The initial energy of each sensor node was $20[\mathrm{~J}]$.

\subsection{Performance criteria}

In this subsection, we describe how to evaluate the fault tolerance. We consider the sensing of a target is successful when at least one sensor node is within sensing range of it and at least one of these nodes has a route to the base station.

In order to evaluate the tolerance against battery exhaustion, we consider the case where the number of sensor nodes that exhaust their battery energy increases as the number of generated targets increases.

As for performance metrics, both the $x \%$-lifetime and the cumulative number of received bits are used. Here, $x \%$ lifetime is defined as the time (i.e., number of targets generated) when the virtual sensing-success-ratio drops below $x \%$. The virtual sensing-success-ratio means the probability that sensing a target is successful in the current state. Here, we say "virtual", because it does not mean the actual sensing-success-ratio but the probability of sensing is successful if a target is generated in the current state. It is calculated after target sensing is completed.

Cumulative number of received bits is incremented when the base station receives new data (note that we increment them only once for he same data though multiple sensor nodes send the same data). A longer lifetime means higher tolerance against battery exhaustion and more cumulative received bits means a lower ratio of missing the target. In each simulation, 200 targets were generated and the simulation was repeated five times. 
In order to evaluate the tolerance against random failure, we considered the case where each node fails with the probability of $p$. The sensing-success-ratio was evaluated for various values of $p$. In each simulation, 1000 targets were generated and the simulation was repeated 30 times. The performance metrics was the the virtual sensing-successratio.

\section{Simulation Results}

\subsection{Tolerance against battery exhaustion}

To show the transient behavior, we plot the number of cumulative received bits in Fig. 5. On the left, they are lowest for simple diffusion. As the number of objects increased, the number of received bits in constant placement saturated first. The number of received bits was higher in R-random placement in almost all regions.

Figure 6 shows 5\%-lifetime and the number of cumulative received bits corresponding to the lifetime. R-random placement had the longest lifetime and the most received bits.

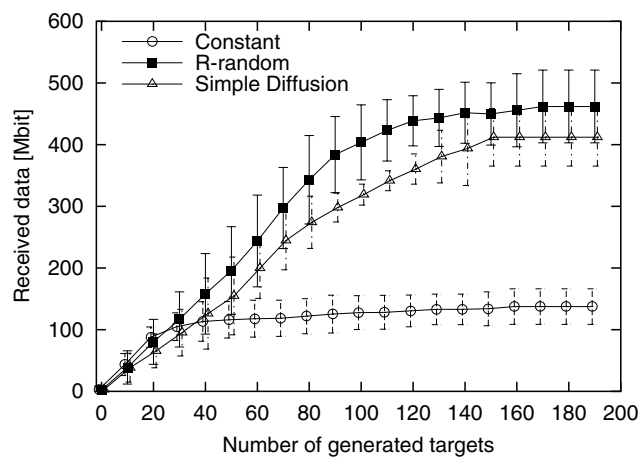

Figure 5. Cumulative received bits.

These results can be explained as follows. Since sensors nearer the base station have a higher probability of being used as relay nodes, these nodes exhaust their battery energy earlier. Therefore, the lifetime becomes longer when the density nearer the base station is higher. However, in that case, there is a higher probability of there being no sensors in the sensing range, which decreases cumulative received bits.

In order to apply the explanation to the results, the probability of there being no sensors within sensing range of a target is shown in Fig. 7 and the conditional probability of there being no routes (when there are sensors that can sense) is shown in Fig 8. In addition, Fig. 9 shows the number of alive nodes (i.e, nodes that did not exhaust their battery energy) within $x[\mathrm{~m}]$ of the base station. In Fig. 7, simple dif-

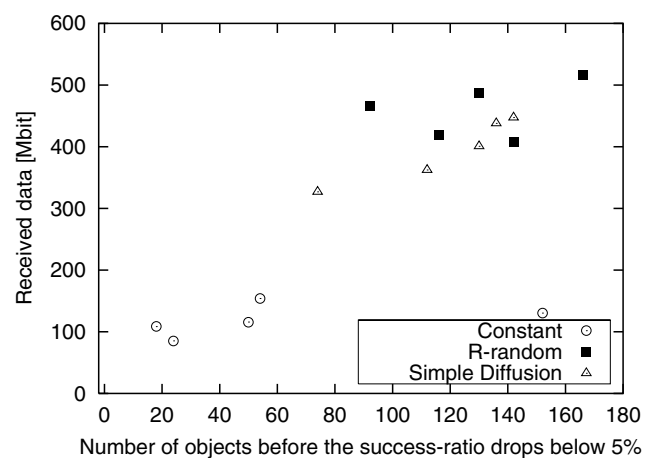

Figure 6. 5\%-lifetime and cumulative received bits.

fusion has the highest probability of having no sensors in any regions, while constant placement has the highest probability of having no routes in Fig. 8. In Fig. 8, the number of alive nodes nearer the base station is much lower, especially for simple diffusion and constant placement. That is why the probability of there being sensors within sensing range is high and the lifetime is long in R-random placement.

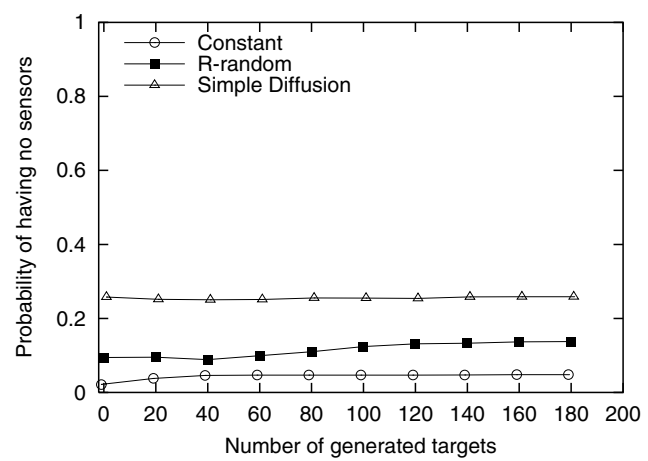

Figure 7. Probability of having no sensors.

\subsection{Tolerance against random failure}

The sensing-success-ratio is shown in Fig. 10. When the sensor error ratio is low, simple diffusion has the lowest success-ratio and constant placement has the highest. This is because the probability of there being sensor nodes that can sense is lowest in simple diffusion and highest in constant placement. However, when the sensor error ratio exceeds 0.4 , the success-ratio in constant placement drops rapidly, which means that the fault tolerance in constant placement 


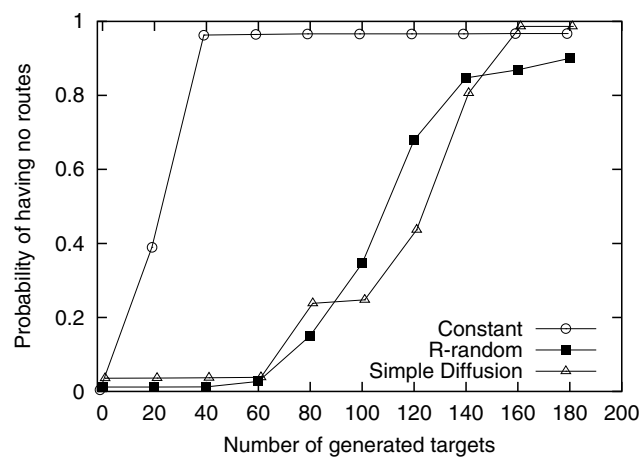

Figure 8. Conditional probability of having no routes.

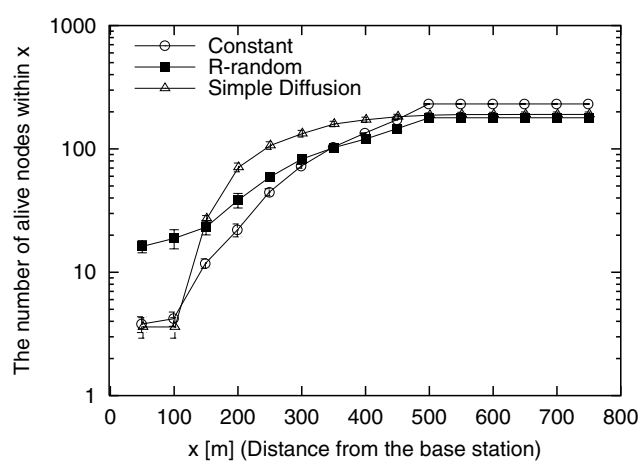

Figure 9. The number of alive nodes within $x[\mathrm{~m}]$ of the base station

is the lowest of the three. In all regions, R-random shows a higher success-ratio.

From another point of view, evaluation on tolerance against random failure is equivalent to that on the density of sensor nodes in region $A$. Considering this, Fig. 10 also indicates success-ratio for R-random placement is almost proportional to the number of sensor nodes in region $A$, while this is not true in the other placements. We consider this observation reveals the essential advantage of Rrandom placement in that the newly added nodes are effectively placed when the number of nodes increases. In other words, R-random placement well brings out the potential of sensor nodes, which leads to the desirable performance of the sensor network.

In order to examine the results in detail, we plot the probability of having no sensors in Fig. 11 and the conditional probability of having no routes (when there are sensors that can sense) is shown in Fig. 12. As shown in Fig. 11, the probability of there being no sensors that can sense does not decrease as the error ratio decreases in simple diffusion, while it decreases in constant and R-random placement. As for routes to the base station, constant placement shows much higher probability of there being no routes in Fig. 12. Summarizing above, we can say R-random placement has a higher probability of having both nodes that can sense and routes to the base station.

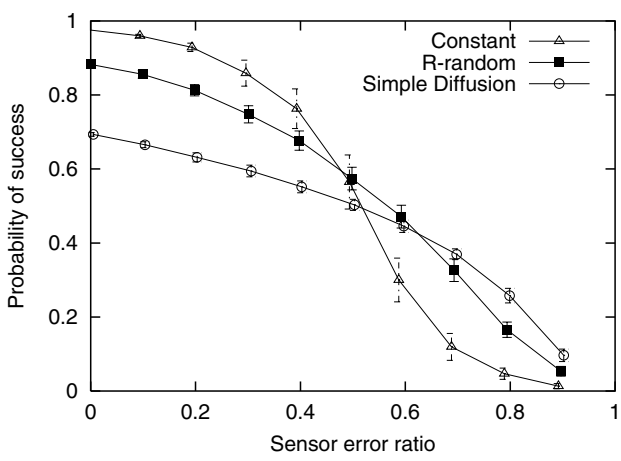

Figure 10. Sensing-success-ratio.

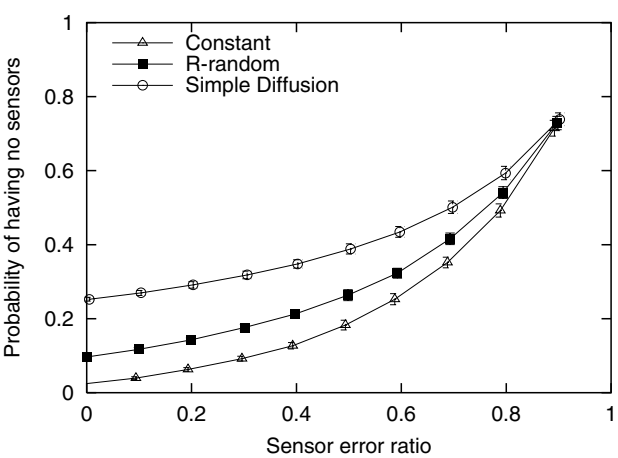

Figure 11. Probability of having no sensors.

\section{Conclusion}

In this paper, we evaluated the fault tolerance in sensor networks and examined how to place sensor nodes to raise the tolerance. Through simulation study, we found a trade-off between the existence of nodes that can sense and the tolerance against failure. Simple diffusion and constant placement are the most typical stochastic placements. However, results show that the probability of having nodes that can sense is low in simple diffusion and the tolerance against failure is low in constant placement. R-random placement, which is our proposal, has high fault tolerance 


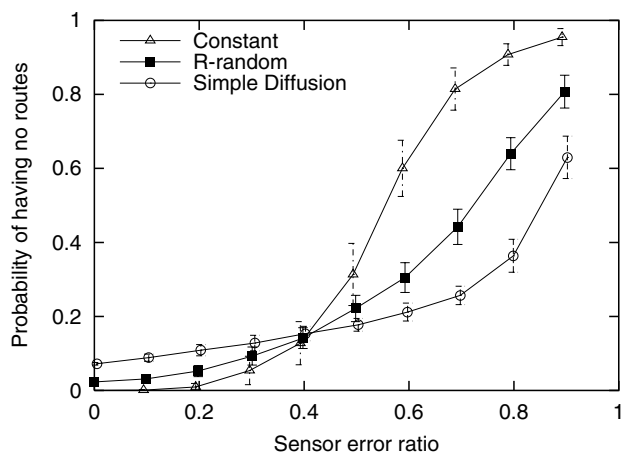

Figure 12. Conditional probability of having no routes.

with the high probability of having nodes that can sense. This is because the density near the base station is higher, while that far from the base station is not too low.

R-random placement may not be optimal, but the results in this paper indicate that the fault tolerance against both battery exhaustion and random failure can be raised by improving sensor-placement and R-random placement is an example of good placement. Moreover, the results also shows R-random placement well brings out the potential of sensor nodes, in that the newly added nodes are effectively placed when the number of nodes increases.

For further study, we plan to establish optimal stochastic placement based on the observations reported in this paper.

\section{Acknowledegment}

We would like to thank Mr. Keisuke Ishibashi for his valuable comments and Ms. Kyoko Ashitagawa for her help with the simulations.

\section{References}

[1] I. F. Akyildiz, W. Su, Y. Sankarasubramaniam, and E. Cayirci. A survey on sensor networks. IEEE Соттиnications Magazine, 40(8):102-114, August 2002.

[2] M. Bhardwaj, T. Garnett, and P. Chndrakasan. Upper bounds on the lifetime of sensor networks. In Proc. IEEE ICC '01, pages 785-790, June 2001.

[3] W. R. Heinzelman, A. Chandrakasan, and H. Balakrishnan. Energy-efficient communication protocol for wireless microsensor networks. In Proc. IEEE Hawai Int'l Conf. on System Sciences, pages 3005-3014, January 2000.

[4] W. R. Heinzelman, J.Kulik, and H. Balakrishnan. Adaptive protocols for information dissemination in wireless sensor networks. In Proc. ACM MobiCom '99, pages 174-185, August 99.
[5] G. Hoblos, M. Staroswiecki, and A. Aitouche. Optimal design of fault tolerant sensor networks. In Proc. IEEE Int'l Conference on Control Applications, pages 462-472, September 2000.

[6] C. Intangonwiwat, R. Govindan, and D. Estrin. Directed diffusion: A scalable and robust communication paradigm for sensor networks. In Proc. ACM MobiCom '00, pages 56-67, August 2000.

[7] L. Li and Y. Halpern. Minimum-energy mobile wireless networks revisited. In Proc. IEEE ICC '01, pages 278-283, June 2001.

[8] T. Poggio and S. Edelman. A network that learn to recognize three-dimensional objects. Nature, 343(6255):263-266, January 1990.

[9] E. Shih, S. H. Cho, N. Ickes, R. Min, A. Sinha, A. Wang, and A. Chandrakasan. Physical layer driven protocol and algorithm design for energy-efficient wireless sensor networks. In Proc. ACM MobiCom '01, pages 272-286, July 2001.

[10] K. Sohrabi, V. A. J. Gao, and G. J. Pottie. Protocols for selforganization of a wireless sensor network. IEEE Personal Communications, 7(7):16-27, October 2000.

[11] A. Woo and D. Culler. A transmission control scheme for media access in sensor networks. In Proc. ACM MobiCom '01, pages 221-235, July 2001. 